\title{
PSEUDO-RANDOM MODULATION OF A LASER DIODE FOR GENERATING
}

\section{ULTRASONIC LONGITUDINAL WAVES}

\author{
Eric I. Madaras and Robert F. Anastasi* \\ * U.S. Army Vehicle Technology Center, ARL, AMSRL-VT-S \\ NASA Langley Research Center \\ Mail Stop 231 \\ Hampton, VA 23681-2199
}

\section{INTRODUCTION}

Laser generated ultrasound systems have historically been more complicated and expensive than conventional piezoelectric based systems, and this fact has relegated the acceptance of laser based systems to niche applications for which piezoelectric based systems are less suitable. Lowering system costs, while improving throughput, increasing ultrasound signal levels, and improving signal-to-noise are goals which will help increase the general acceptance of laser based ultrasound.

One current limitation with conventional laser generated ultrasound is a material's damage threshold limit. Increasing the optical power to generate more signal eventually damages the material being tested due to rapid, high heating [1,2]. Generation limitations for laser based ultrasound suggests the use of pulse modulation techniques as an alternate generation method. Pulse modulation techniques can spread the laser energy over time or space, thus reducing laser power densities and minimizing damage. Previous experiments by various organizations using spatial or temporal pulse modulation [3-9] have been shown to generate detectable surface, plate, and bulk ultrasonic waves with narrow frequency bandwidths . Using narrow frequency bandwidths improved signal detectability, but required the use of expensive and powerful lasers and opto-electronic systems.

The use of a laser diode to generate ultrasound is attractive because of its low cost, small size, light weight, simple optics and modulation capability. The use of pulse compression techniques should allow certain types of laser diodes to produce usable ultrasonic signals. The method also does not need to be limited to narrow frequency bandwidths. The method demonstrated here uses a low power laser diode $(\sim 150 \mathrm{~mW})$ that is modulated by controlling the diode's drive current and the resulting signal is recovered by cross correlation. A potential application for this system which is briefly demonstrated is in detecting signals in thick composite materials where attenuation is high and signal amplitude and bandwidth are at a premium. 


\section{EXPERIMENT}

The heart of the experimental set up was a laser diode that operated at a wavelength of $809 \mathrm{~nm}$. The CW output power was measured to be $160 \mathrm{~mW}$ and the diode could be modulated with a bandwidth of up to $1 \mathrm{GHz}$. The diode had a threshold turn on current of 23 $\mathrm{mA}$ and a full operating current of $230 \mathrm{~mA}$. The beam characteristics after passing through an aspherical collimating lens was a beam with dimensions of approximately 2 by $6 \mathrm{~mm}$ and a Gaussian cross section. It was estimated that the beam focal size approached $8 \mu \mathrm{m}$ after focusing with a $75 \mathrm{~mm}$ focal length lens. This would produce a maximum power density for the $\mathrm{CW}$ beam at the focus on the order of $\sim 10^{5} \mathrm{~W} / \mathrm{cm}^{2}$.

For generating ultrasonic signals, either pseudo-random or frequency modulated chirp sequences were used. The pseudo-random sequence consisted of 2048 pulses of variable temporal length light pulses and was generated following MacWilliams, et. al. [10] The code length for the total sequence was $2.3 \mathrm{~ms}$, and it covered a bandwidth from 0.1 to $\sim 1.6 \mathrm{MHz}$. The chirp sequences consisted of varying the diode's output amplitude with a sinusoidal chirp of total length of $2 \mathrm{~ms}$. The chirp covered a bandwidth from either 0.1 to $1 \mathrm{MHz}$ or 0.5 to $1.5 \mathrm{MHz}$. The drive signal for the laser diode was provided by a laser diode driver that could modulate its output range a full $200 \mathrm{~mA}$. In addition, an offset current could be set to compensate for the threshold current of the diode. The pulse rise and fall times were measured to be less than $10 \mathrm{~ns}$. The signal to drive the laser driver was provided by an arbitrary waveform generator that operated at $100 \mathrm{MHz}$ with $400 \mathrm{Ms} / \mathrm{s}$ and 8 bit amplitude control. It had two output channels with $1 \mathrm{MB}$ of memory per channel.

The measurement set-up, in figure 1, shows the basic through transmission test configuration. On reception, we used a $12.5 \mathrm{~mm}$ diameter, 0.5 or $1.0 \mathrm{MHz}$ piezoelectric contact transducer. The transducer signal was amplified with either a 40 or $60 \mathrm{~dB}$ ultrasound pre-amplifier. Signals from the pre-amplifier were digitized using the computer's ADC card at 8 bits resolution, digitizing at either 6.25 or $12.5 \mathrm{MHz}$ rates. Signal averaging from 10 to 100 signals helped to stabilize against baseline variations after which a cross correlation was performed between the drive signal and the receive signal. A background signal was also captured following the same procedures except with the beam blocked. Subtraction of the background signal removed radiated transmitter noise emanating from the diode driver.

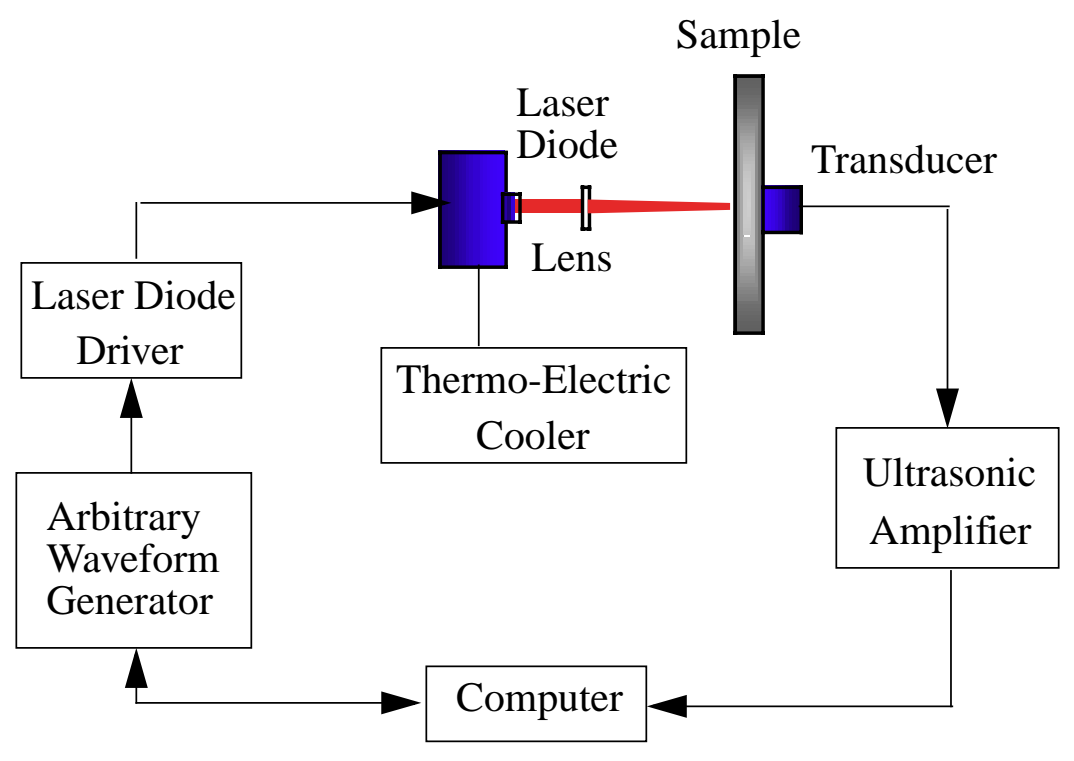

Figure 1. The experimental measurement apparatus. 


\section{MEASUREMENTS AND RESULTS}

Figure 2 illustrates a comparison of the transmitted pseudo-random sequence, the received signal and the resulting correlation signal. Ultrasound was generated on a flat aluminum plate with a nominal thickness of $12.5 \mathrm{~mm}$ and detected with a $0.5 \mathrm{MHz}$ transducer. Figure 2a shows a portion of on-off, variable length, pseudo-random code, and Figure $2 \mathrm{~b}$ shows the received signal for the same time segment, illustrating how little ultrasonic signal is generated directly. Figure $2 \mathrm{c}$ shows the resulting signal after correlation of the transmit and received signals. The estimated first arrival of the longitudinal wave is indicated by the arrow in the figure. It is evident that the signal is arriving at the indicated moment, followed by shear waves and multiple reverberations.

The comparison of several different generation methods is made in Figure 3. Figure 3a shows a conventional pitch-catch signal using a $0.5 \mathrm{MHz}$ longitudinal mode transducer for both transmission and reception. Figure $3 \mathrm{~b}$ illustrates the signal that is generated by a Qswitched laser delivering a $4 \mathrm{~mJ}$ pulse of light, $50 \mathrm{~ns}$ long and using a $0.5 \mathrm{MHz}$ longitudinal

a)

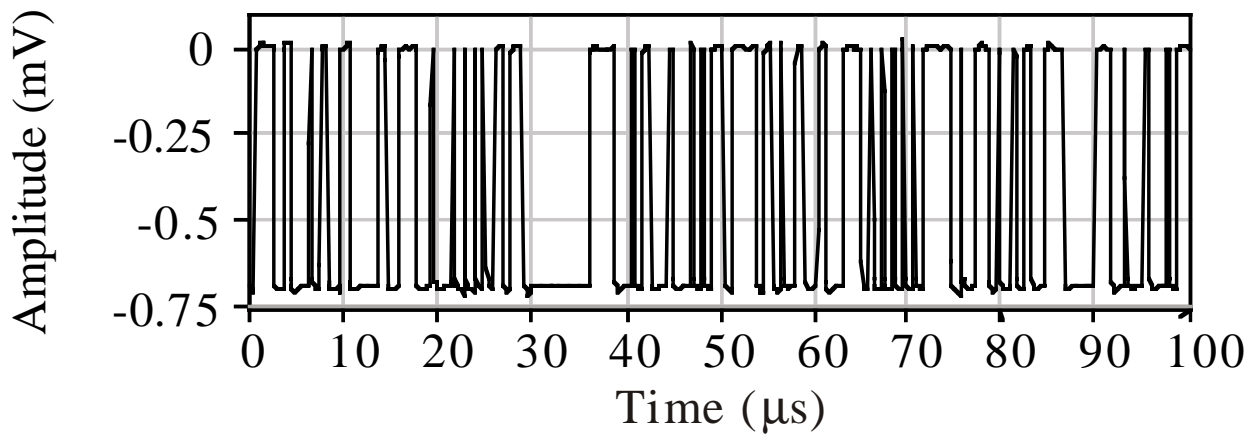

b)

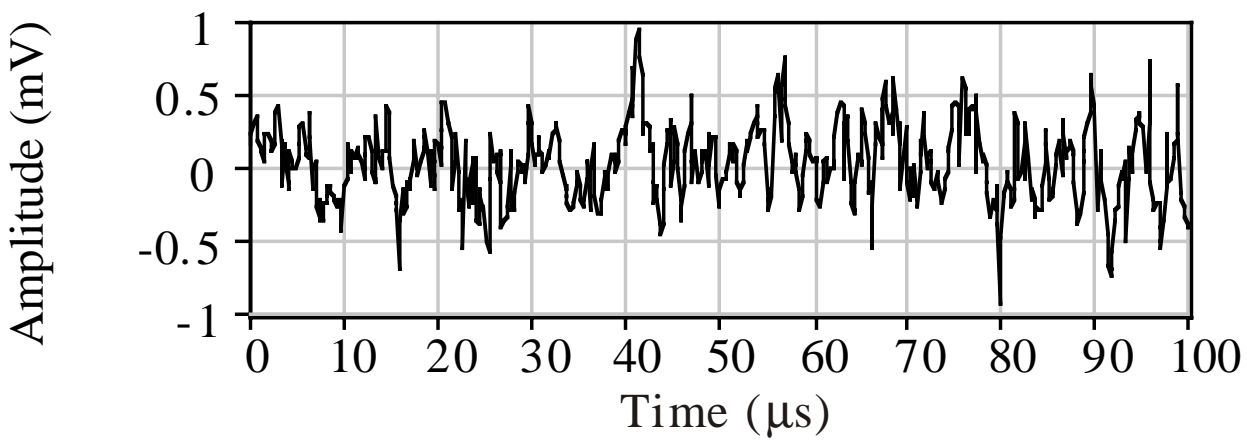

c)

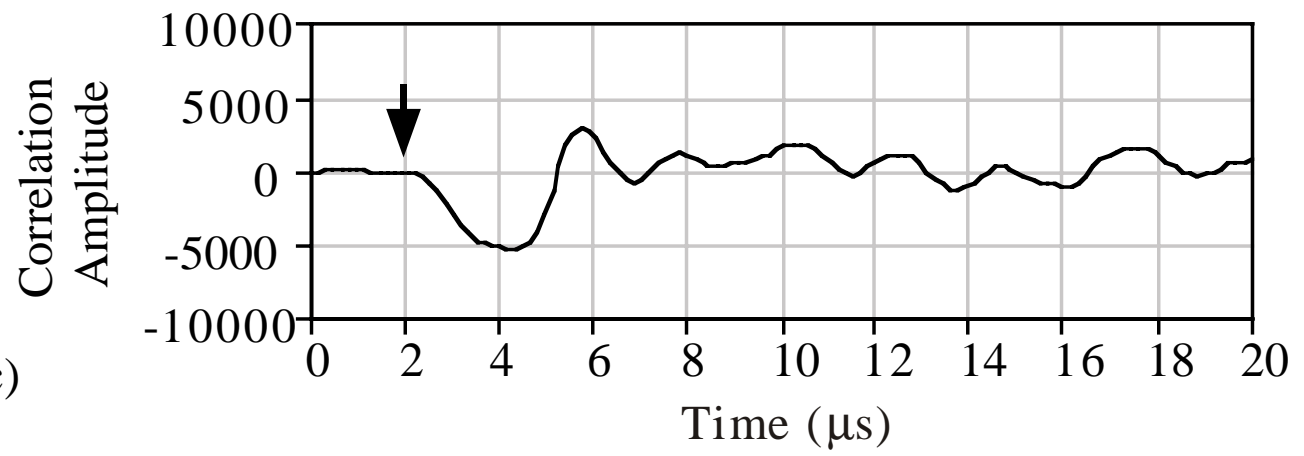

Figure 2. A comparison of portions of the transmitted, received and resulting correlation signals. a) A portion of the transmitted pseudo-random code. b) The received signal captured at the same moment as panel a). c) The cross correlation output. The arrow indicates the estimated time of arrival of the longitudinal wave. 
a)

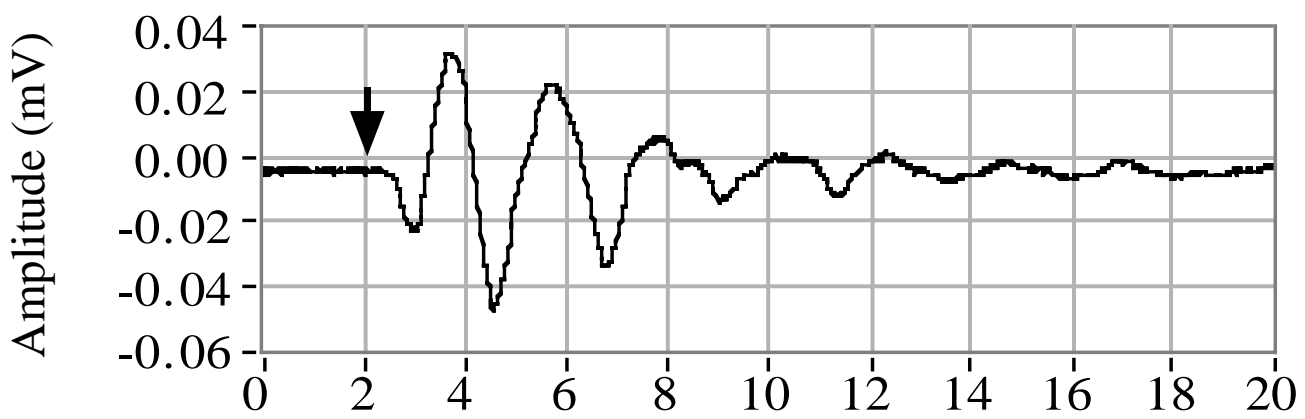

b)
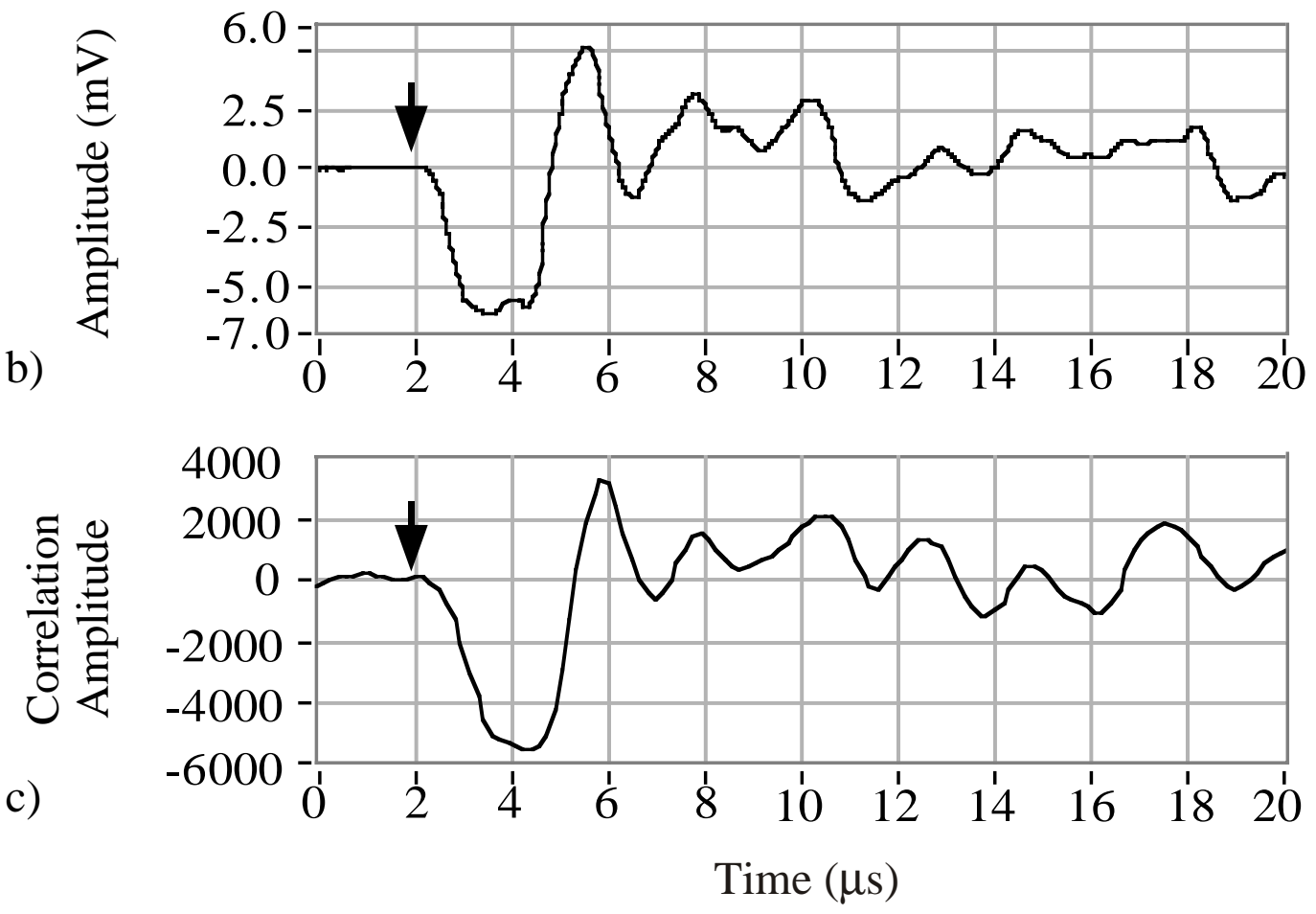

Figure 3. Comparison of different generation methods. a) Shows the signal from using piezoelectric transducers for generation and reception. b) Shows the signal from using a Q-switched laser pulse for generation. c) Shows the signal from using the pulse modulated laser diode for generation. The arrows in each panel indicate the estimated first arrival time predicted from the published speed of sound in aluminum.

mode transducer for reception. Figure $3 \mathrm{c}$ shows the signal generated with the modulated laser diode and is the same signal shown in figure $2 \mathrm{c}$. In the case of figure $3 \mathrm{c}$, the pulse code delivered about $0.16 \mathrm{~mJ}$ of energy, approximately twenty-five times less energy than used to generate figure $3 \mathrm{~b}$. In figure 3 , it is clear that the first signal's arrival is the same for all generation mechanisms. The arrows in all three panels indicate the computed first arrival time using the published value for the speed of sound in aluminum. Figure 3a illustrates the common ringing signal seen with electro-mechanical generation. Since a longitudinal transducer is being used, the signals should be longitudinal, and the first reverberation should start interfering with the original signal near the six microsecond point in time and the echoes should repeat about every four microseconds. In the cases for the laser generated signals, both longitudinal and shear waves will be produced. The longitudinal wave appears near two microseconds. The shear wave should appear after about four microseconds, which is seen in the figures $3 \mathrm{~b}$ and $3 \mathrm{c}$ as a distortion in the wave and interrupts the signal's upward trend at that time. The laser generated signals illustrate strong correlations in the wave patterns between the two methods. It is also interesting to note that the signal to noise ratios for the two laser generations methods appear similar. 
In figure 4, we have an example of signals that are generated in a composite sample. This composite sample was a composite plate, $13.7 \mathrm{~mm}$ thick with through thickness stitches. The plate had several flat bottom holes milled into the plate to produce a series of different thicknesses; $4.3 \mathrm{~mm}, 6.6 \mathrm{~mm}$, and $8.6 \mathrm{~mm}$ as well as the full plate thickness. Previous measurements on this composite were reported [11]. The velocity was measured to be $3480 \mathrm{~m} / \mathrm{s}$. The arrows in figure 4 indicate the anticipated first arrival of the longitudinal wave for each thickness, (a) $1.24 \mu \mathrm{s}$, (b) $1.90 \mu \mathrm{s}$, (c) $2.48 \mu \mathrm{s}$, and (d) $3.94 \mu \mathrm{s}$. It is evident in the figures that the signals are indeed arriving near the predicted time. In the case of the thinner samples seen in figure $4 \mathrm{a}$ and $4 \mathrm{~b}$, the longitudinal and shear signals are not resolved, but for the thicker samples, figure $4 \mathrm{c}$ and $4 \mathrm{~d}$, the waves forms are separating.

One obvious issue with using pulse modulation methods is the potential advantage of controlling the bandwidth of the signal. Q-switched lasers generate sound over a large bandwidth, a feature which is difficult to control. In the case of thick or attenuating materials, much of that signal is not usable. If the energy could be directed into usable

a)

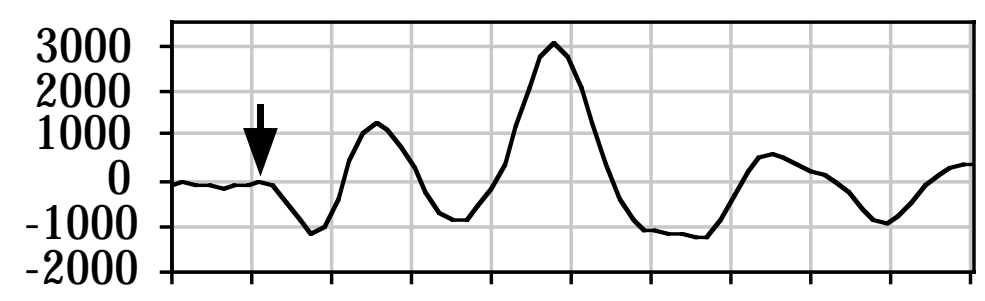

b)

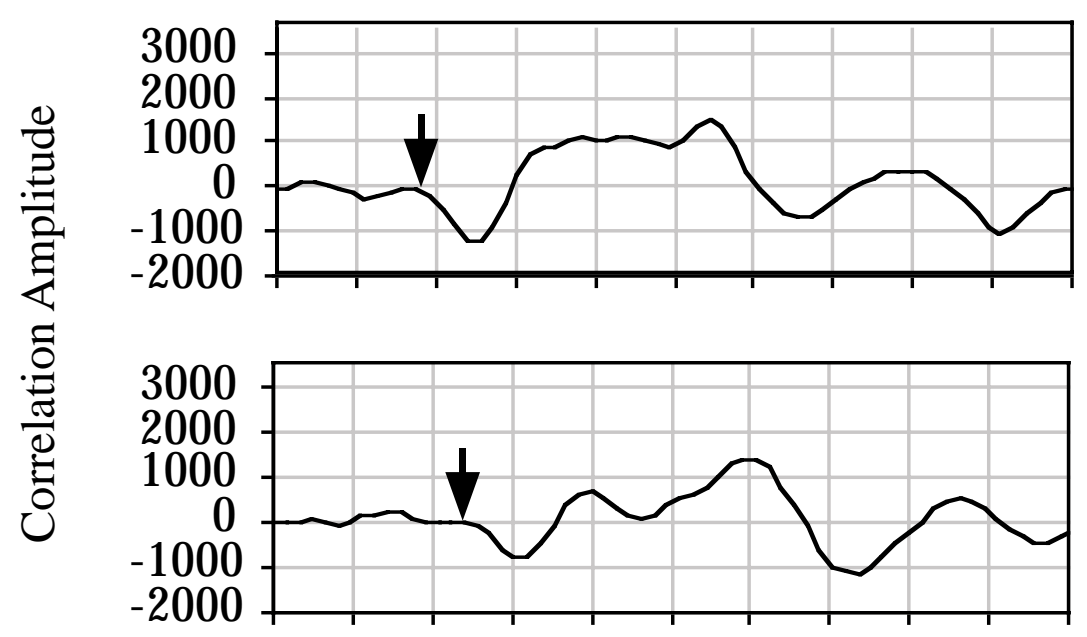

c)

d)

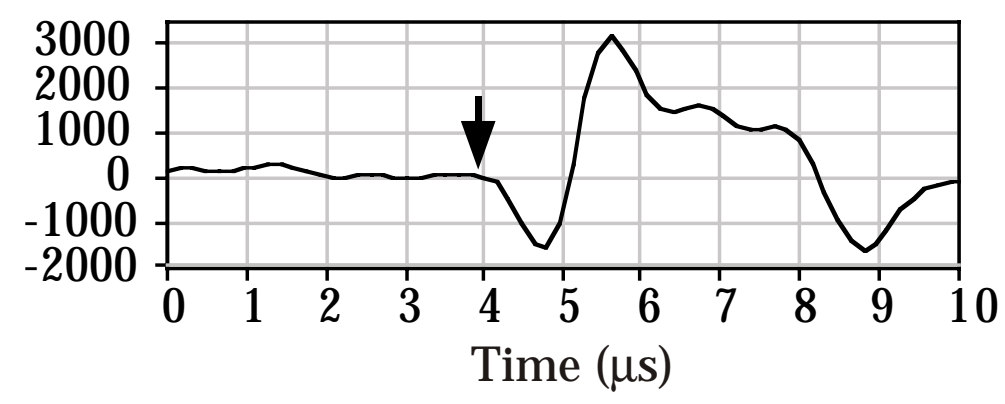

Figure 4. Comparison of the generation of ultrasound in composites using a pseudo-random code with a laser diode. a) Shows the signal from a $4.3 \mathrm{~mm}$ thick composite. b) Shows the signal from a $6.6 \mathrm{~mm}$ thick composite. c) Shows the signal from a $8.6 \mathrm{~mm}$ thick composite. d) Shows the signal from a $13.7 \mathrm{~mm}$ thick composite. The arrows in each panel indicate the estimated time of arrival for the longitudinal wave. 
frequencies, some efficiency might be realized. One straight foward method to test the capability to control the bandwidth of laser diode generation is to use a chirp signal. The frequency of interest can be quickly and easily controlled with such a generation code. In figure 5, different generation bandwidths are compared. In figure 5a, a chirp signal covering the bandwidth of 0.1 to $1.0 \mathrm{MHz}$ is used to drive the diode while the detection is made with a $0.5 \mathrm{MHz}$ transducer, effectively matching the band pass of the drive signal to the transducer. Figure $5 \mathrm{~b}$ represents a chirp with a bandwidth of 0.5 to $1.5 \mathrm{MHz}$ with a $1.0 \mathrm{MHz}$ detection transducer, again matching the laser drive signal with the transducer's bandwidth. Figure $5 \mathrm{c}$ shows the case where the bandwidth of the transmission, 0.5 to $1.5 \mathrm{MHz}$, is not matched by using a $0.5 \mathrm{MHz}$ detection transducer. It is evident that in panel b) the frequency of the signal is very near one $\mathrm{MHz}$, and that the signal is at a higher frequency than in panel a). At the $1 \mathrm{MHz}$ frequency, the wave patterns are starting to become temporally resolved. In figure $5 \mathrm{c}$, the chirp is set to only partially overlap the transducer's bandwidth. Under such conditions, it would be expected that a weaker signal would be detected and that it would be of an intermediary frequency. Inspection of figure $5 \mathrm{c}$ shows both features.

a)

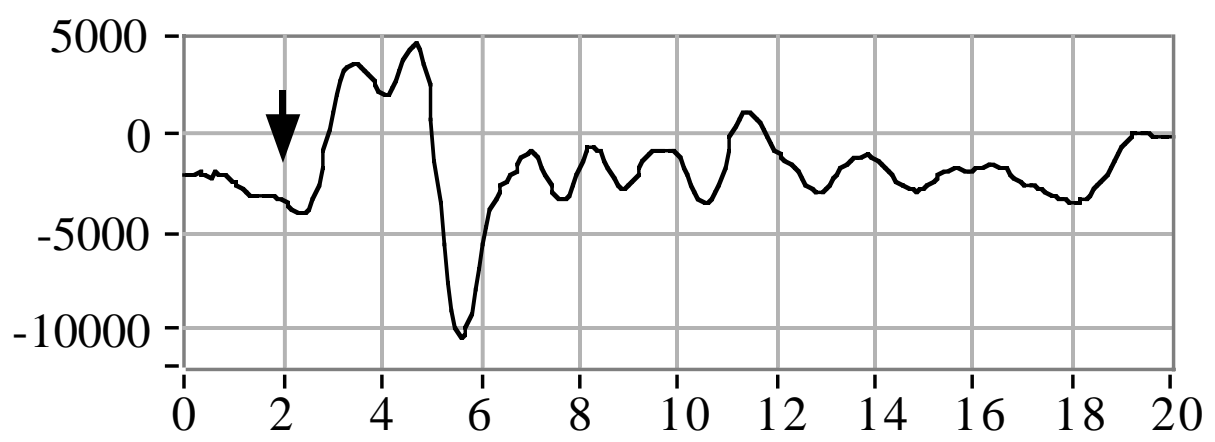

b)

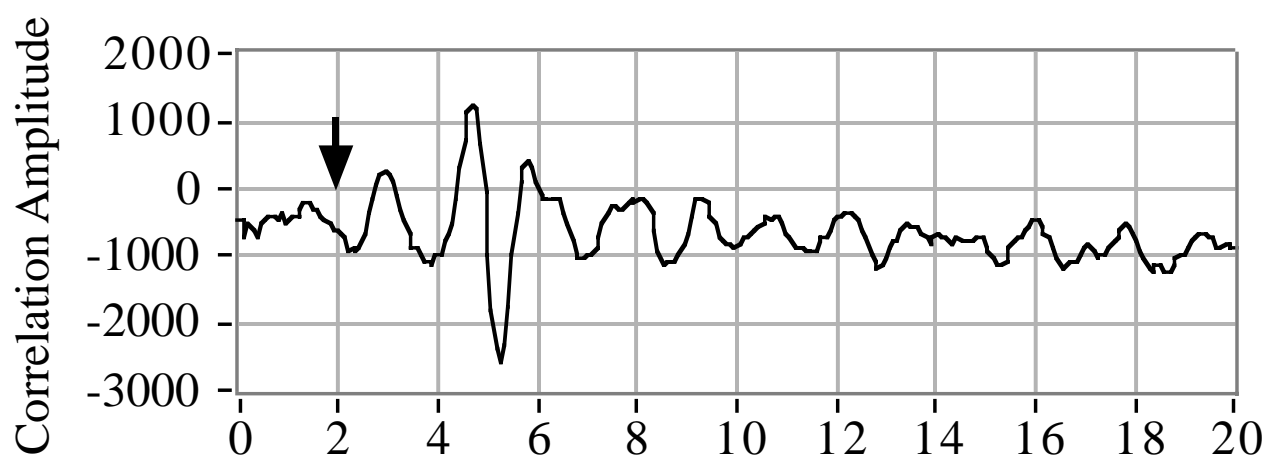

c)

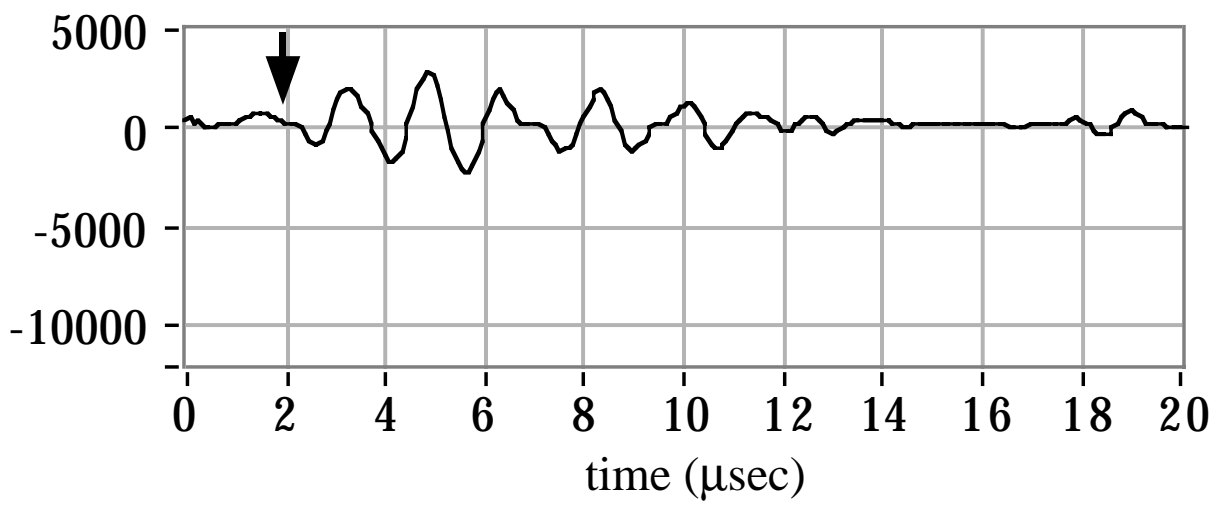

Figure 5. Comparison of several different generation and reception bandwidths. a) Shows the signal from using a 0.1 to $1.0 \mathrm{MHz}$ chirp and a $0.5 \mathrm{MHz}$ piezoelectric transducer. b) Shows the signal from using a 0.5 to $1.5 \mathrm{MHz}$ chirp and a $1.0 \mathrm{MHz}$ piezoelectric transducer. c) Shows the signal from using a 0.5 to $1.5 \mathrm{MHz}$ chirp, but with a $0.5 \mathrm{MHz}$ piezoelectric transducer 


\section{DISCUSSION}

These results illustrate the successful generation of ultrasonic bulk waves in aluminum and composite samples using pulse compression methods with a modulated laser diode without damaging the test material. In the simple examples shown here, the ultrasonic signals obtained with the modulated laser diode compared well to those obtained using conventional through transmission and to Q-switched laser generated signals. The laser-generated signals exhibited similar signal to noise ratio even though the laser diode generated signal was obtained with 25 times less excitation power than the Q-switched laser generated signal. The laser-diode power density was estimated to be a factor of 10 to 1000 below the material's damage threshold, and the ultrasound generated from a single diode laser pulse could not be viewed directly on an oscilloscope. In principle, greater signal to noise levels can be realized by using longer pulse compression codes or, since the excitation power density was well below the material damage threshold, by using more powerful laser diodes as they become available. Finally, it has been shown that signal bandwidth can be controlled. This is a desirable trait that is lacking in more conventional laser based methods. The ability to control the ultrasound signal to noise ratio and bandwidth electronically is an extremely desirable feature for nondestructive evaluation applications, especially when coupled with using a very small, economical, compact, non-contacting excitation probe.

\section{REFERENCES}

1. C.B Scruby and L.E. Drain, Laser Ultrasonics: Techniques and Applications, Adam Hilger, New York, 1990

2. D.A. Hutchins, "Ultrasonic Generation by Pulsed Lasers," Physical Acoustics. Vol. 18,1988

3. R. K. Ing, F. Gires, and M. Fink, "Focusing and Beam Steering of Laser Generated Ultrasound," Ultrasonics Symposium Proceedings, vol. 1, 1989

4. A. D. McKie, J. W. Wagner, J. B. Spicer, and C. M. Penney, "Laser Generation of Narrow Band Directed Ultrasound," Ultrasonics, vol. 1. 27, n. 6, pp. 323-330, 1989.

5. L. Wang, J. S. Steckenrider, and, J. D. Achenback, "A Fiber-Based Laser Ultrasonic System for Remote Inspection of Limited Access Components," Rev. of Prog. In Quan. Nondestructive Eval., vol. 16A,

6. S. G. Pierce, B. Culshaw, and, Q. Shan, "Laser Generation of Ultrasound Using a Modulated Continuous Wave Laser Diode," Appl. Phys. Letters,

7. R. Pierce, C. Ume, and J. Jarzynski, "Temporal Modulation of a Laser Source for the Generation of Ultrasonic Waves," Ultrasonics, Vol. 33, No. 2, 1995

8. J. W. Wagner, J.B. Deaton Jr., and J.B. Spicer, "Generation of Ultrasound by Repetitively Q-Switching a Pulsed Laser,” Applied Optics, Vol. 27, No. 22, November 1988

9. J. B. Deaton, A.D.W. McKie, J.B. Spicer, and J.W. Wagner, "Generation of NarrowBand Ultrasound with Long Cavity Mode-Locked Nd:YAG Laser,” Appl. Phy. Lett., 56 (24) 11 June 1990

10. F. J. MacWilliams and N.J.A. Sloane, "Pseudo-Random Sequences and Arrays," Proceedings of the IEEE, Vol. 64, No. 12 December 1976.

11. R. F. Anastasi, A.D. Friedman, M.K. Hinders, and E.I. Madaras, "NDE of Damage in Thick Stitched Composites Using Laser-Based Ultrasound with Optical Fibers," Materials Evaluation, Vol. 56, No. 12, December 1998 